\title{
Pesticide Use Trends in the U.S.: Pesticides for Industrial/Commercial/Governmental Uses ${ }^{1}$
}

Frederick M. Fishel ${ }^{2}$

\section{Introduction}

The EPA, in cooperation with the USDA and FDA, is responsible for regulating the production and use of pesticides in the U.S. This document is one of a series that provides data on volumes used and sales of pesticides from the latest EPA survey data available, 2001 - 2002. This document focuses on pesticides used by the industrial/commercial/governmental market sector. Other documents within this series address the agricultural and home and garden sectors. The intent of this information is only to present an objective profile and does not attempt to interpret, reach conclusions about, or make inferences regarding the data. Conclusions should not be drawn in regards to impacts on human health, the environment, or the economy.

\section{Data sources}

The data reported in this document are based upon EPA estimates. EPA does not have a program devoted specifically to estimating pesticide use; 
classes of pesticides - herbicides, insecticides, fungicides, and other pesticides. The "herbicide" data combine plant growth regulators (PGR) with them, while "fungicides" and "insecticides" exclude sulfur and petroleum oil. Data summarized for "other" pesticides combine the total for rodenticides, molluscicides, nematicides, fumigants, aquatic, pesticides for the control of birds and fish, and other miscellaneous pesticides. Sulfur and petroleum oil are included in the "other" category as well. The use data shown in Table 2 are presented similarly, except that nematicides and fumigants are presented as a separate category. The EPA survey use data exclude industrial wood preservatives, sulfur, petroleum oil, specialty biocides, chlorine/hypochlorites, and other chemicals used as pesticides. In reporting the amount used, data are presented as pounds of active ingredient (a.i.). Totals may not add precisely due to rounding.

\section{U.S. industrial/commercial/governmental pesticide expenditures}

Total expenditures for industrial/commercial/governmental pesticide use as a whole were similar each year (Table 1). U.S. industrial/commercial/governmental pesticide expenditures totaled an average of more than $\$ 1.5$ billion averaged over 2000 and 2001. Expenditures on herbicides/plant growth regulators accounted for the largest portion of total expenditures - more than $50 \%$ both years, followed by expenditures on insecticides and miticides, fungicides, and other pesticides, respectively. There was little change in relative quantities of pesticide expenditures for each class of pesticide between years.

\section{U.S. industrial/commercial/governmental pesticide amount used}

U.S. industrial/commercial/governmental pesticide amount used in both 2000 and 2001 was more than 100 million pounds averaged over the 2 years (Table 2). The largest portion of total U.S. industrial/commercial/governmental pesticides used each year was herbicides, followed by nematicides and fumigants, fungicides, insecticides and miticides, and other pesticides. Total volume of

industrial/commercial/governmental pesticides used was similar each year.

\section{Most commonly used conventional industrial/commercial/governmental pesticide active ingredients}

Table 3 shows the ten most commonly used conventional industrial/commercial/governmental pesticide active ingredients in 2001 compared with 1999. 2,4-D was the most-used active ingredient during both years, between 16 million and 20 million pounds. The four most commonly used active ingredients use rankings were consistent for both years. Of the top ten active ingredients, six were herbicides; two each were fungicides and insecticides. Due to the fact that some applicators in this sector may also apply pesticides in the home and garden sector, there may be some usage reported in one market that may have occurred in the other.

\section{Additional information}

Kiely, T., D. Donaldson, and A. Grube. 2004. Pesticides Industry Sales and Usage: 2000 and 2001 Market Estimates. EPA's Biological and Economic Analysis Division, Office of Pesticide Programs, and Office of Prevention, Pesticides, and Toxic Substances http://www.epa.gov/pesticides. 
Table 1. U.S. industrial/commercial/governmental pesticide expenditures by pesticide class -2000 and 2001 .

\begin{tabular}{||l|c|c||}
\hline \hline Class & \multicolumn{2}{|c||}{ Millions \$ } \\
\hline $\mathbf{2 0 0 0}$ & 762 & 51 \\
\hline Herbicides/PGR & 468 & 32 \\
\hline Insecticides/Miticides & 172 & 11 \\
\hline Fungicides & 83 & 6 \\
\hline Other & 1,485 & \\
\hline Total & & 52 \\
\hline $\mathbf{2 0 0 1}$ & 792 & 33 \\
\hline Herbicides & 510 & 11 \\
\hline Insecticides/Miticides & 172 & 4 \\
\hline Fungicides & 61 & \\
\hline Other & 1,535 & \\
\hline Total & & \\
\hline \hline
\end{tabular}

Table 2. U.S. industrial/commercial/governmental pesticide amount used by pesticide class -2000 and 2001 .

\begin{tabular}{||l|c|c||}
\hline \hline Class & Millions Pounds a.i. & \% of Total \\
\hline $\mathbf{2 0 0 0}$ & 48 & 42 \\
\hline Herbicides/PGR & 17 & 15 \\
\hline Insecticides/Miticides & 19 & 17 \\
\hline Fungicides & 24 & 21 \\
\hline Nematicides/Fumigants & 6 & 5 \\
\hline Other & 114 & \\
\hline Total & & 44 \\
\hline 2001 & 49 & 14 \\
\hline Herbicides & 15 & 17 \\
\hline Insecticides/Miticides & 19 & 22 \\
\hline Fungicides & 24 & 3 \\
\hline Nematicides/Fumigants & 4 & \\
\hline Other & 111 & \\
\hline Total & & \\
\hline \hline
\end{tabular}

Table 3. Ten most commonly used conventional industrial/commercial/governmental pesticide active ingredients (millions pounds active ingredient).

\begin{tabular}{||l|c|c|c|c|c||}
\hline \hline \multirow{2}{*}{ Active Ingredient } & \multirow{2}{*}{ Type $^{*}$} & \multicolumn{2}{|c|}{$\mathbf{2 0 0 1}$} & \multicolumn{2}{|c||}{1999} \\
\cline { 2 - 6 } & & Rank & Range $^{\text {** }}$ & Rank & Range \\
\hline 2,4-D & $\mathrm{H}$ & 1 & $16-18$ & 1 & $17-20$ \\
\hline Glyphosate & $\mathrm{H}$ & 2 & $13-15$ & 2 & $11-14$ \\
\hline Copper sulfate & $\mathrm{F}$ & 3 & $4-6$ & 3 & $5-7$ \\
\hline Pendimethalin & $\mathrm{H}$ & 4 & $3-5$ & 4 & $3-5$ \\
\hline Chlorothalonil & $\mathrm{F}$ & 5 & $2-4$ & 7 & $2-4$ \\
\hline Chlorpyrifos & $\mathrm{I}$ & 6 & $2-4$ & 5 & $3-5$ \\
\hline Diuron & $\mathrm{H}$ & 7 & $2-4$ & 8 & $2-4$ \\
\hline \hline
\end{tabular}


Table 3. Ten most commonly used conventional industrial/commercial/governmental pesticide active ingredients (millions pounds active ingredient).

\begin{tabular}{|c|c|c|c|c|c|}
\hline \multirow[t]{2}{*}{ Active Ingredient } & \multirow[t]{2}{*}{ Type } & \multicolumn{2}{|c|}{2001} & \multicolumn{2}{|c|}{1999} \\
\hline & & Rank & Range $^{\star \star}$ & Rank & Range \\
\hline MSMA & $\mathrm{H}$ & 8 & $2-4$ & 6 & $2-4$ \\
\hline Triclopyr & $\mathrm{H}$ & 9 & $1-3$ & 10 & $1-3$ \\
\hline Malathion & 1 & 10 & $1-3$ & 9 & $1-3$ \\
\hline
\end{tabular}

